\title{
Problems and Integrating Procedure of Quadcopter with Anemometer for Collect Wind Velocity Data
}

\author{
Azamataufiq Budiprasojo ${ }^{1, *}$, Bayu Rudiyanto ${ }^{1}$, Budi Hariono $^{2}$, and Tosawat Seetawan ${ }^{3}$ \\ ${ }^{1}$ Department of Engineering, Politeknik Negeri Jember, Jl. Mastrip 164, \\ Jember 68101, Indonesia \\ ${ }^{2}$ Department of Agriculture Technology, Politeknik Negeri Jember, Jl. Mastrip 164, \\ Jember 68101, Indonesia \\ ${ }^{3}$ Faculty of Science and Technology, Sakhon Nakhon Rajabhat University, \\ Sakon Nakhon 47000, Thailand
}

\begin{abstract}
Wind speed profiles are generally sought by using an anemometer. However, problems occur when taking wind speed data over large areas, such as mapping the potential of wind energy in someplace. Altitude and coordinate variations will vary and difficult to executed if using a conventional anemometer. In this modern era where drones, which are unmanned Aerial Vehicles (UAVs) are increasingly used, have the prospect of being used as a tool in retrieving data. A type of drone that has an economical price is a quadcopter type drone. Integrating this drone with an anemometer will then be able to retrieve wind speed data at the coordinates and elevation. The wind speed survey process will be more accurate and can be saved in terms of cost and time. Problems and procedures in integrating these two tools will be studied in this study because both of these tools have specific characteristics such as turbulence from propeller drones that can interfere with data accuracy and sensitivity problems to the sensor anemometer that requires special attention. The development of quadcopter, including construction, modifying, and tuning, will be main issues to discuss in this journal.
\end{abstract}

Keywords: renewable energy, survey with quadcopter type drone, wind energy

\section{Introduction}

Quadcopter drone is a type of drone that has relatively more usage compared to other types of drones. This type of drone is widely used because it is easier to use, compact in size and

\footnotetext{
${ }^{*}$ Corresponding author: azamataufiq@polije.ac.id
} 
has a more competitive price. The naming of the quadcopter drone is because it has four arms equipped with a propeller on each arm [1-2].

The use of quadcopter drones began to penetrate the research field, especially in the field of survey research and mapping. One research survey that has a relationship with drones is a survey about the potential of wind energy [1-4].

This survey was conducted as an initial activity in planning a wind power plant. Wind speed data becomes a reference in making plans for the location of generators, windmill designs and the potential for electrical energy that can be generated by power plants. Wind power plants need to get intense development so that according to Indonesian government policies that begin to diversify electricity sources from fossil fuels to alternative energy sources.

By using drones as survey aids, the data collection process is expected to be faster and more accurate. Drones can reach several observation points with variations in height and distance [5]. Currently, drones with special designation as surveying tools are produced at relatively high prices, for example, 3DR's Site Scan UAS can cost over USD 10000.

With a specific designation only taking wind speed data at certain coordinates, of course, there is a desire to make a drone with essential features and can be used more widely. Take, for example, the price of a quadcopter drone with GPS features and being able to carry a load of about $1 \mathrm{~kg}$ costing only costs USD 400. To build an anemometer data logger it only costs USD 150 . So, for total, it only costs about USD 550, it is much cheaper than buying a USD 10000 surveyor drone [6-8].

Problems and procedures in integrating these two tools will be studied in this study because both of these tools have specific characteristics such as turbulence from propeller drones that can interfere with data accuracy and sensitivity problems to the sensor anemometer that requires special attention.

\section{Initial requirements}

Figures and tables, as originals of good quality and well contrasted, are to be in their final the initial need for a survey system are that integrating the drone with a measuring instrument, which in this case is an anemometer type. This problem occurs when conducting a survey of wind energy potential on Payangan Beach in Jember, Indonesia.

Because of the large area that has to be surveyed and the contours of hilly land with uneven flat roads, it will be difficult to force to retrieve data using a regular anemometer. The logical solution to solve this problem is to utilize a drone that is integrated with an anemometer measuring instrument which then becomes a new survey tool that can be called a wind velocity surveyor drone [9].

Through this research, Researchers are determined some problem to solve such as:

(i). The prototype must be easy to use without needing advanced skills,

(ii). The prototype must collect accurate data in a large area with variation of coordinate and elevation, and no interrupted by drone propeller air turbulence,

(iii). The prototype must safe to use and have a good durability,

(iv). The prototype must be easily retrievable after each flight,

(v). The prototype must be able to conduct multiple flights,

(vi). The prototype must be cheap and have a easy to get parts to replacing with fast if there were a trouble or broke,

(vii). The prototype must be able to collect data near real time at least one data every second,

(viii). The prototype must be able to flight about $10 \mathrm{~min}$ and carry load about $500 \mathrm{~g}$. 
Table 1. Required accuracies for wind speed data.

\begin{tabular}{|l|l|}
\hline Variable & Accuracy \\
\hline Surround Air Temperature & $\pm 0.5^{\circ} \mathrm{C}$ \\
\hline Specific Air Humidity & $\pm 0.25 \mathrm{~g} \mathrm{~kg}^{-1} \mathrm{H}_{2} \mathrm{O}$ \\
\hline Wind Direction & $\pm 25^{\circ}$ from datum \\
\hline Wind Speed & $\pm 0.1 \mathrm{~m} \mathrm{~s}^{-1}$ \\
\hline Air Pressure & $\pm 0.5 \mathrm{kPa}$ \\
\hline
\end{tabular}

Because this surveyor drone is a prototype that is modified from quadcopter drone equipped with an anemometer, therefore, two tools become the main component to select first.

\subsection{Anemometer}

Researchers considered various type of anemometer sensor, such using a hotwire anemometer, a cup anemometer. From research observation, it is conclusion that a hotwire anemometer is more capable to measure wind velocity data if integrated it in quadcopter drone.

Measured wind data above rapidly spinning rotors anemometer is more complicated. The blades create a downwash wind turbulence and could possibly affect the airflow around the anemometer. The rotor effects create a drag force going bigger as the copter is descending fly off.

The hot-wire anemometer, is an anemometer equipped with a hotwire sensor to collect wind velocity data, which works by heating a small wire and measuring the convective heat loss as a result of ambient airflow, is traditionally used in more delicate settings to measure highly turbulent flows. It requires frequent recalibration due to dust accumulation but has a rapid response time. It does not capture wind direction, however, and a separate wind vane would be required, if not multiple anemometers.

Beyond simply selecting the appropriate measurement device, it also needed to determine the appropriate distance to place the anemometer from the rotors to avoid turbulence in airflow or false readings. Although researchers expect cleaner data as the anemometer is further from the propellers, the increased moment arm of a larger support shaft also decreases the stability of the copter.

In order to test for the appropriate anemometer distance, researchers brought the copter to a large room with no ventilation to ensure the test would not be affected by unsteady wind. The copter was mounted on a shaft roughly 10 feet off the ground in the center of the room to minimize ground effects from the props. The anemometer was attached on three differently sized mounts $-40 \mathrm{~cm}$ to $+40 \mathrm{~cm}$ with $10 \mathrm{~cm}$ differences between nodes. The $0 \mathrm{~cm}$ node is for center of propeller. Researchers then collect wind data while alternating between props on and props off once every minute. 


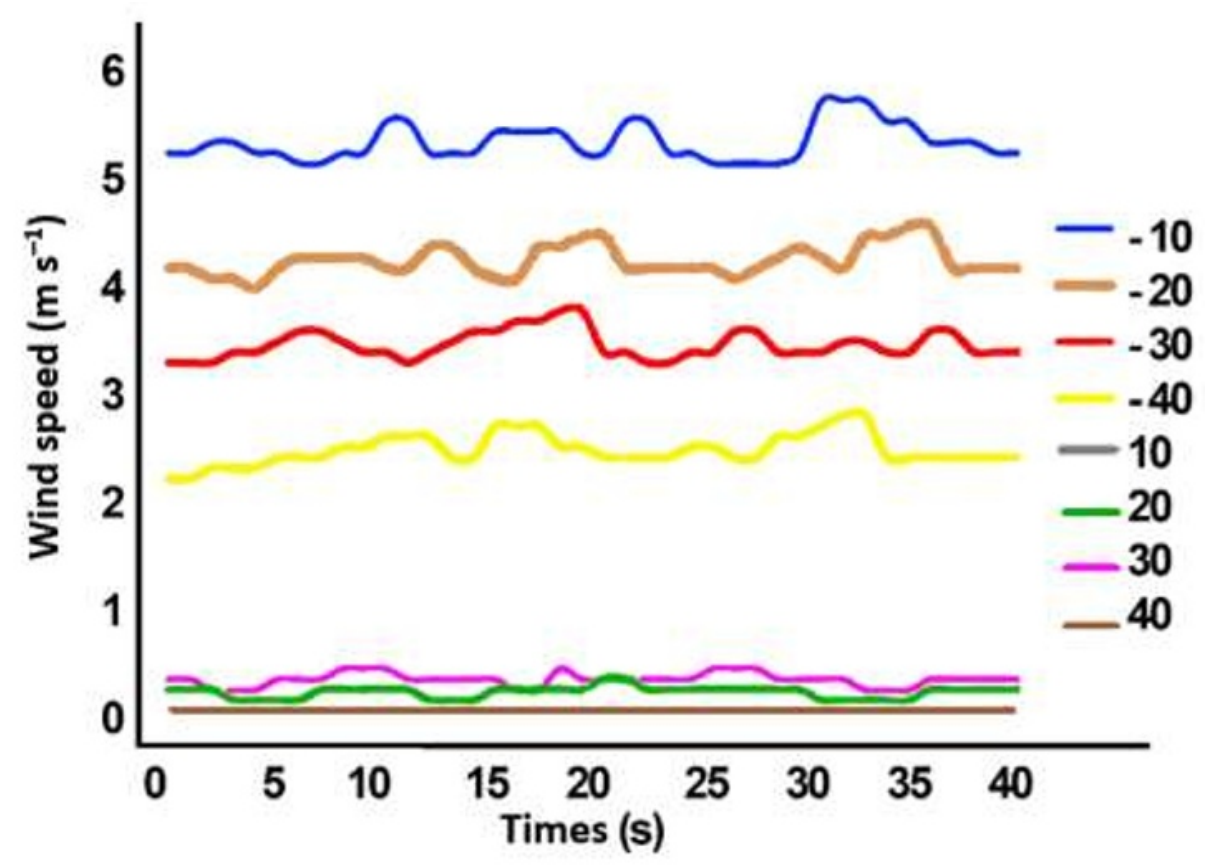

Fig. 1. Graph of wind velocity.

Graph in Figure 1 is about wind velocity data takes under and above the propeller, straight to propeller edges, with propeller speed rotation holding in maximum speed. Hot wire sensor places face direct to wind flows. The graph legends show the distance from the center of the propeller as a datum.

From the graphs in Figure 1. There is fact if the sensor is positioned right at the edges of the propeller and propeller is turned on with an idle speed of $1154 \mathrm{rpm}(1 \mathrm{rpm}=1 / 60 \mathrm{~Hz})$. For the direct sensor position, the safe position to place sensor is above the propeller with minimum distance $30 \mathrm{~cm}$ above the propeller. All sensor positioned below the propeller will be disturbed by propeller wind. For indirect sensor position, the safe position to place sensor is above propeller with minimum distance $10 \mathrm{~cm}$ above the propeller. If sensor placed below the propeller then the safe position is at $20 \mathrm{~cm}$ below the propeller.

It can be concluded that the safeties place to places sensor is above the propeller at the edges of the drone propeller or at the center of the drone. But If the sensor placed at the center of the drone, it must be attention that at that area, there is a meet zone of four propeller. It can cause a vortex zone and can reduce the accuracy of the sensor. So, researchers prefer to place the sensor at the side of drone to avoid the sensor to get disturb by chaotic vortex.

For real time data recording, researchers choose to make an anemometer data logger with hot wire win sensor, and it's had a memory card (MMC) component to record and save the data. For attention anemometer data logger with PC connected cable for saving data is must be avoiding because its complexity and extra weight when users want to place it into the drones.

For anemometer type it's can use an Arduino Uno microcontroller as a main base of anemometer MMC data logger or market version anemometer. For Arduino or other own fabricating microcontroller, researchers must carefully calibrate it with calibrate licensed standard anemometer. For easy of fabrication and modifying, researchers suggest using a market version anemometer. It can easily modify and no worry about its accuracy because its already calibrate certified. 


\subsection{Drone}

As the initial requirements researchers must make numerous design and decisions of the prototype. Researchers began by selecting a drone on which became the base this prototype. Researchers decided to build prototype from a multirotor drone since it would be smaller, easier to take off, and offer more stability rather than airplane drone. But this research also considers while a multirotor is also constrained by limited of battery life to support power. So, it must select with carefully which type of multirotor drone match with requirement [10]. In fact, that more rotors are added, greater stability and lift are gained but at the other hand its increased battery usage and cost.

Multi rotors come in various shapes and designs; the most common are the four-rotor who commonly known as quadcopter. The quadcopter configuration was selected as it provided sufficient lift to carry the expected payload and offered security against motor failure.

From preliminary flight testing, researchers found the battery life to be roughly $10 \mathrm{~min}$ in low-wind conditions, allowing enough time for data collection. However, the battery life is subject to change based upon wind condition and flight plan. Currently, the copter is controlled by remote with a feature to turn back home and following the pilot with usage of GPS.
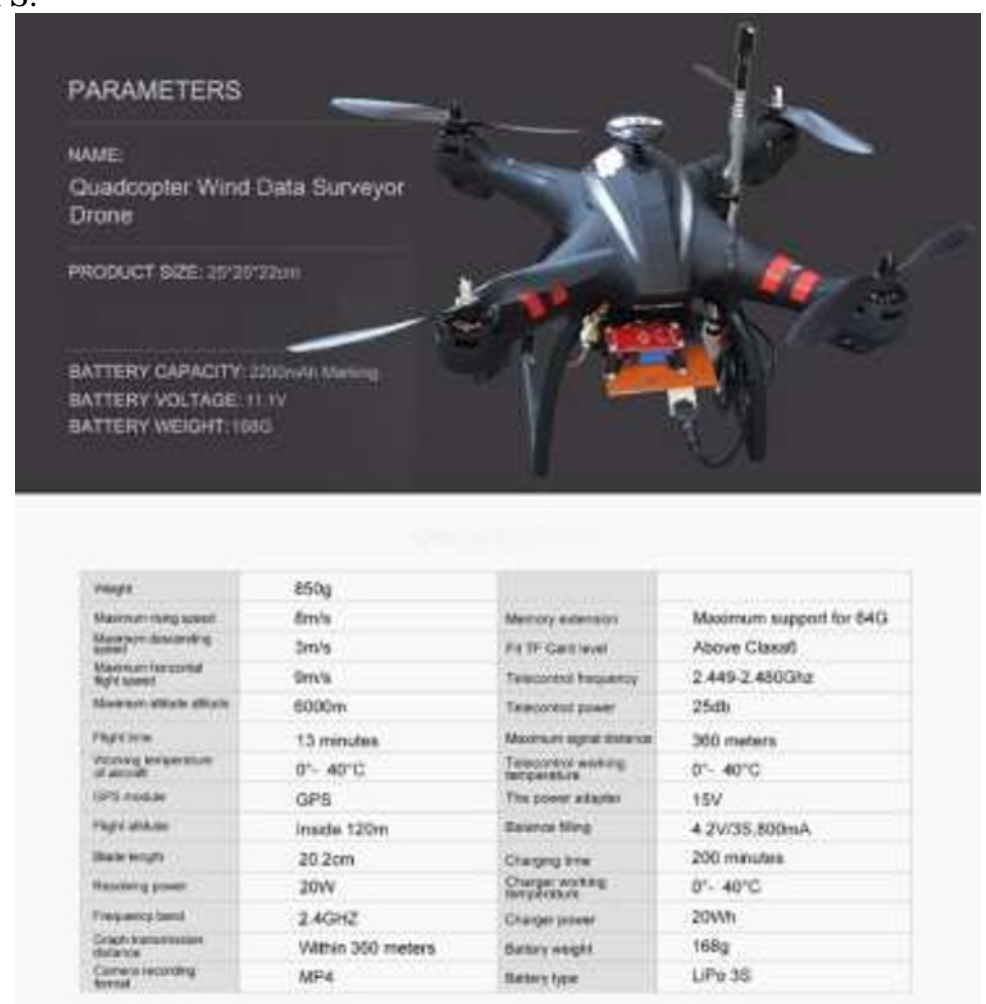

Fig. 2. Quadcopter parameters.

As quadcopter parameter fact, it fair enough to say that its accomplish some initial requirement. But for extend its ability to flight much longer with load carry and turbulence and air drag resistant, researchers must anticipate by double the battery capacity but precisely same in dimension with old battery. Researchers also remove some component such a gimbal and camera to light weight the drone and make some space to put 
anemometer tools. This Quadcopter now have the ability to carry load about $1 \mathrm{~kg}$ and can fly much longer.

\subsection{Integrating procedure}

The anemometer must be placed below the drone to avoid it instability because of central gravity changing. The Lower center of gravity placed in a drone, the more stable drone flight. The anemometer server is a cantilevered weight, so the fact that the higher its placed, the less stable the copter becomes. On the other hand, the best location to place the anemometer server is above the rotors or It can say below the drone. It is desired to minimize the effect of rotor downwash on the wind velocity data.

Users must place the anemometer in a board and screw it on a gimbal holder below the drone. Carefully and make sure it screws in a center of the drone. Users surely don't want it in tilted position because it can make the propeller to work harder to stabilize the drone when flight.

For anemometer power supply users can get the power from drone battery. This process can save a weight of the prototype. Because the differences between the drone battery voltage output and current are different from anemometer Input voltage, users cannot just connect cable from drone battery to anemometer battery. The drone battery is $12 \mathrm{~V}$ and the anemometer battery is only $6 \mathrm{~V}$. Users must add some resistor to compliance the problem. In this prototype, reserachers use a regulator to step down the voltage from $11.1 \mathrm{~V}$ output from drone battery to $9 \mathrm{~V}$ for anemometer input voltage. This process success step down the voltage of the drone battery to safe anemometer input voltage.

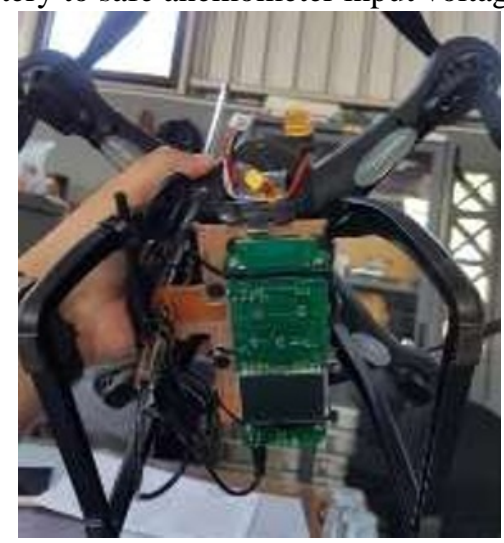

Fig. 3. Integrated anemometer below the drone.

For hot wire sensor position, it should place at the top of the drone and propeller, as already know in this area is not affected by propeller wind. The sensor must place directly forward to the wind flow.

The anemometer was placed $30 \mathrm{~cm}$ above the copter propeller due to constraints in copter stability. At this length, the propeller has zero effect on the recorded wind speed. Real-time data will be stored on an MMC SD card mounted on the board. Furthermore, the individual sensors will be easily connected to the board through individual pins or a serial connection.

The real-time data is a raw data and numerically presented to the user in excel format. This data can then be uploaded to a computer and run through the same Microsoft office excel application for further analysis. Figure 4 shows the complete flow diagram for the system. The following picture is a flow diagram in the modification of the drone. There are 
three lines found on the drone to classify electric lines, data lines and radio or wireless lines.

Electrical lines are lines in the form of cables that need to be connected. data path is the path where data from the wind speed is processed. Of course, in this data path there is also an electrical relationship, but don't show it to simplify the schema. Wireless lines are a path that displays the process of sending and receiving radio waves between the receiver that is on the drone and the transmitter held by the user.

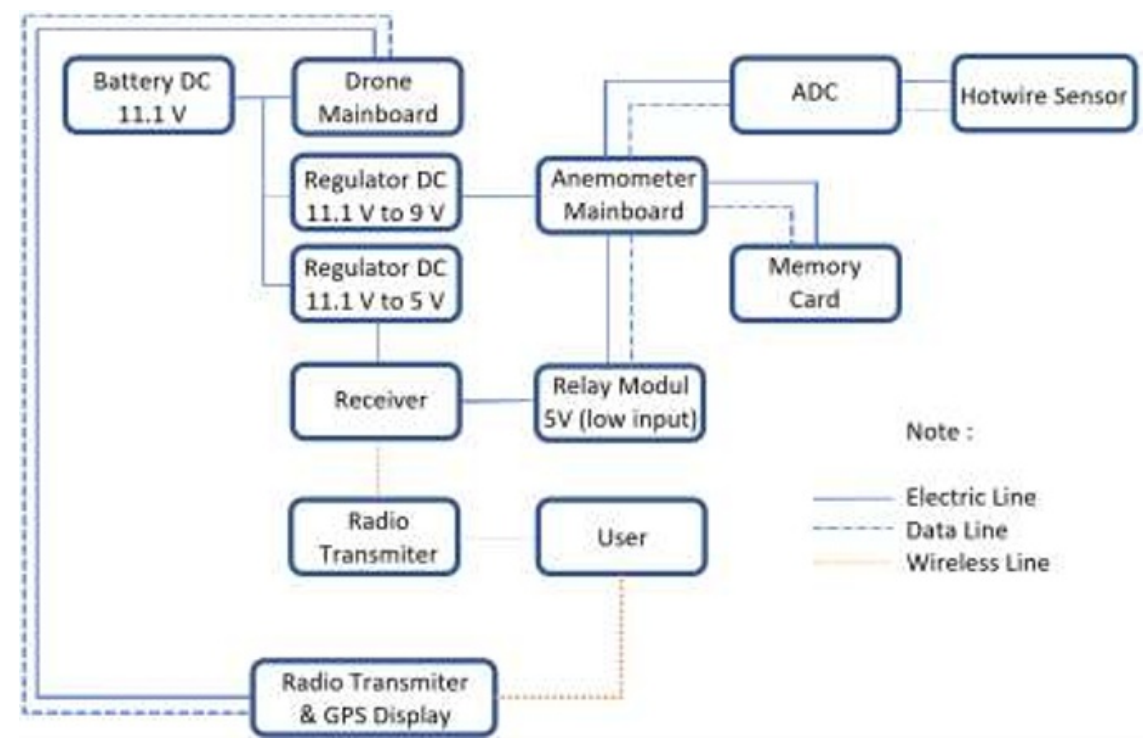

Fig. 4. Flow diagram of anemometer surveyor drone.

Researchers want the specific the drone to only take data when the drone is at the point of coordinates and elevation. This needs to be done to make it easier for us to identify data when the data has been imported to the computer. By taking data shortly after the drone has stabilized at the desired coordinates and elevation, identification of the data can be easily done through recorded time records.

The solution is to add transmitters and receivers to these devices as seen at Figure 5. Receiver is attached to the anemometer which makes the new anemometer record data when the receivers command it. a transmitter in the form of a remote will be operated to control the command to retrieve wind speed data only when users want the data to be recorded. 


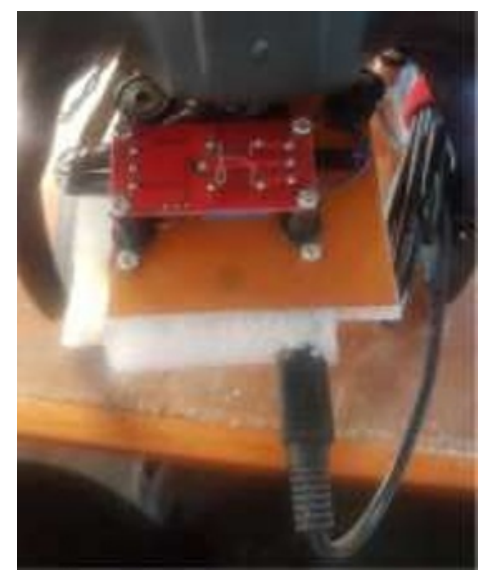

Fig. 5. Radio receiver added to anemometer for precise time data recording.

This data recording remote control receiver for data retrieval control, requires a voltage of $5 \mathrm{~V}$ and is taken from the drone battery after being reduced from $11.1 \mathrm{~V}$ to $5 \mathrm{~V}$ using a DC regulator.

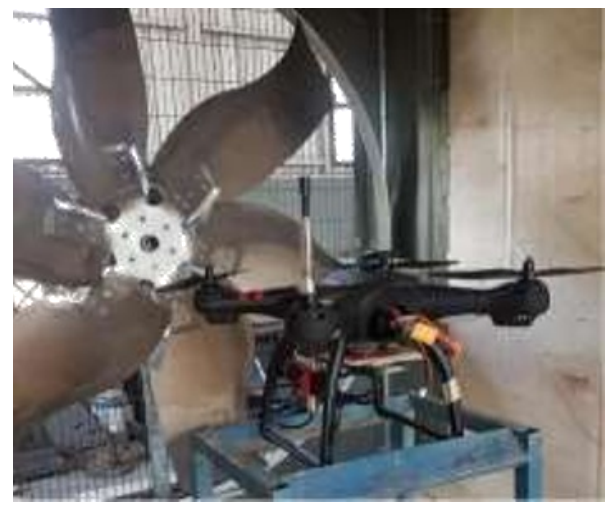

Fig. 6. Complete prototype of anemometer quadcopter surveyor drone.

\section{Results}

The anemometer quadcopter surveyor drone platform was able to retrieve data at every second. The required accuracies for the sensors were met. The velocity sensor can collect data within the $\pm 0.1 \mathrm{~m} \mathrm{~s}^{-1}$. The data successfully recorded to MMC SD Card and can download to the personal computer.

When testing the anemometer, there are no problems in recording and retrieving data. Problems arise only in the anemometer settings which are reset to the default settings when the drone is turned off. This can happen because the anemometer draws power from the drone's battery.

The solution to this problem is adding two capacitors of 0.5 microfarads to the input power of the anemometer. the addition of this capacitor is successful in preventing anemometer reset to standard conditions if the power of drone is turned off.

Manual flight testing over the lifetime of the sensor payload has demonstrated the anemometer quadcopter surveyor drone's ability to take off and land without damage to the 
sensor payload. The copter is able to deliver a complete data set that can be accessed by the user at the end of a flight.

Researchers also tested the crash simulation with the copter and the result the copter remains in one piece and got no major damage. Nevertheless, in $3^{\text {rd }}$ crush testing It got problem that the propeller become stuck by sand who accidently come to the propeller dynamo through the cooling vent of the quadcopter propeller. However, it without dissemble the copter, it can fix it by blow the compressed air through the van. Suggested adding some filtering layer within the vent to prevent the sand get in.

Through the use of Microsoft excel App the data can converted to charts specifically; wind velocity data can be visualized show the behavior if wind in the selected area in with various scenarios during flights testing. Through flight testing with the sensor and anemometer integrated payload, the quadcopter's is able to support the entire payload of the system, weighing about half a kilogram. The rate during take-off and landing has been set to be controllable by the user.

The anemometer quadcopter surveyor drone's platform has demonstrated its stability in flight through an intricate tuning process. The anemometer quadcopter surveyor drone's tuning accounts for the addition of the sensor payload, allowing the platform to remain stable during flight.In case of complications during flight, fail-safes have been enabled on the anemometer quadcopter surveyor drone's platform. These problems include loss of battery power or GPS signal, and if it occurs, the copter can return to the take-off location because of it turning back home feature.

Researchers were not able to meet the goal altitude of $120 \mathrm{~m}$ as the copter behavior become more unpredictable and uncontrolled. It suspected that the installation of the antenna will impact on the additional wind resistance so that it interferes with the stability of the quadcopter. But from further information Researchers cannot fly the copter to high because were restricted by FAA regulations to fly under $120 \mathrm{~m}$. The maximum altitude success to receive with steady copter is a $100 \mathrm{~m}$. But for high wind velocity, it just only success flight the copter an $80 \mathrm{~m}$ of altitude.

The current copter configuration has just been tuned, meaning it has not completed an autonomous flight yet, only manual. As a result, researchers do not know if the copter can land autonomously. However, other autonomous function such as altitude and position hold work as it should, and the copter is capable of being landed under manual flight. As a result, it expects that further testing of the copter will complete this goal.

\section{Conclusion}

The new prototype system, which is named anemometer quadcopter surveyor drone, can take wind data measurements autonomously and repeatedly, with minimum setup and know-how required. The quadcopter purchase with the only low costs coming from the replacement of cheap and off the self-parts. The sensor is a hotwire anemometer type and placed above the drone at the edge of the drone to prevent propeller turbulence. Data from sensors convert by analogue to digital converter in anemometer mainboard.

The anemometer placed below the drone to maintenance drone stability with low center of gravity changed. Anemometer interfaced using a Linux based chip with supported MMC interfacing, allowing for easy collection of data. The data is then transferred to the personal computer at the ground station. It can use the power of the drone battery for additional components by first reducing the voltage using a regulator. The addition of a radio remote control needs to be done so that the drone is able to collect data exactly when the drone has reached the survey point of coordinates and elevation. To prevent the settings on the 
anemometer are reset to it is standard, the research necessary to add a capacitor on the input power side of the anemometer. The final product is an autonomous, turn-key, modular data collection system that can be easily modified to add more sensors and to execute advanced flight paths.

\section{References}

1. R.S. Gates, K.D. Casey, H. Xin, E.F. Weeler, J.D. Simmons, Transactions of the American Society of Agricultural Engineers, 47,5:1709-1715(2004).

https://elibrary.asabe.org/abstract.asp?aid=17613

2. J. Solberg. Susceptibility of quadcopter flight to turbulence. [Thesis], Master of Science, Cornell University Graduate School, USA (2018).

https://ecommons.cornell.edu/handle/1813/59689

3. S. Yoon, H.C. Lee, T.H. Pulliam, Computational analysis of multi-rotor flows, 54th AIAA Aerospace Sciences Meeting, (San Diego, USA, 2016), p. 1-11. https://arc.aiaa.org/doi/10.2514/6.2016-0812

4. I. De Boisblanc, N. Dodbele, L. Kussmann, R. Mukherji, D. Chestnut, S. Phelps, et al., Designing a hexacopter for the collection of atmospheric flow data, 2014 Systems and Information Engineering Design Symposium (SIEDS), (Charlottesville, VA, USA, 2014), p. 147-152.

https://ieeexplore.ieee.org/document/6829915/authors\#authors

5. S. Allison, H. Bai, B. Jayaraman, Estimating wind velocity with a neural network using quadcopter trajectories, AIAA Scitech 2019 Forum, 1596, (San Diego, California, 2019). https://arc.aiaa.org/doi/abs/10.2514/6.2019-1596

6. R.J. Barthelmie, S.C. Pryor, An integrated approach to offshore wind energy assessment: Great Lakes 3D Wind Experiment (No. DOE-Cornell-0005379), (Ithaca, New York, USA, 2017).

http://www.geo.cornell.edu/eas/PeoplePlaces/Faculty/spryor/DoE_AIATOWEA/index . html

7. A. Bennett, V. Preston, J. Woo, S. Chandra, D. Diggins, R. Chapman, et al., Autonomous vehicles for remote sample collection in difficult conditions: Enabling remote sample collection by marine biologists, 2015 IEEE International Conference on Technologies for Practical Robot Applications (TePRA), (Woburn, MA, USA, 2015), p. 1-6. https://ieeexplore.ieee.org/document/7219660

8. J.X.J. Bannwarth, Z.J. Chen, K.A. Stol, B.A. MacDonald, Disturbance accomodation control for wind rejection of a quadcopter, 2016 International Conference on Unmanned Aircraft Systems (ICUAS), (Arlington, VA, USA, 2016), p. 695-701. https://ieeexplore.ieee.org/document/7502632

9. P. Bruschi, M. Piotto, F. Dell'Agnello, J. Ware, N. Roy, Procedia Engineering, 168:802-805(2016).

https://www.sciencedirect.com/science/article/pii/S1877705816335871

10. T. Luukkonen, Modelling and control of quadcopter. Independent research project in applied mathematics, Espoo: Aalto school, school of science, (2011), p. 1-22.

https://www.researchgate.net/file.PostFileLoader.html?id=576d16ed93553b24b5721a 9a\&assetKey=AS\%3A376462596165634\%401466767085787 